\title{
Brief Description of the Difference between Ginseng and Codonopsis
}

\author{
Wenzhao Zhang
}

Shandong University of Traditional Chinese Medicine, No.16369, Jingshi Road, Lixia District, Jinan

City, Shandong Province. 250011

962728827@qq.com

Keywords: Ginseng; Codonopsis; Morphological characteristics; Pharmacological effects; Clinical application

\begin{abstract}
Although, Ginseng and Codonopsis belonged to different genus, they were similar in thekjh medicinal properties, pharmacological effects and other aspects, and even someone was forward that the ancient Ginseng was today's Codonopsis. As the price of Ginseng was much higher, Codonopsis was often used to take the place of Ginseng in some clinical application. The author consulted the related literatures of Ginseng and Codonopsis in detail, which was published in recent years, and then made a brief discussion of their origin, morphological characteristics, efficacy, usage and dosage, pharmacological action and clinical application. Depending on the discussion, it could illustrate that the ancient Ginseng was not the modern Codonopsis, and provide a reference for clinical application under some circumstances.

Ginseng and Codonopsis belong to Qi-invigorating herbs in Traditional Chinese medicine, which could contribute to good health and tonify spleen and lung Qi. The morphological characteristics of the plants of the two herbs are similar; there are also many similarities of themselves in chemical composition, pharmacological effects and efficacy. It is easier to confuse to cause some mistakes, resulting in unnecessary economic losses. It was suggested that the ancient Ginseng was modern Codonopsis. This argument reflects that the Ginseng and Codonopsis are difficult to distinguish. For the treatment of some certain symptoms, Codonopsis can be used to replace the more precious and expensive Ginseng. As a result, it is especially important to clear the differences between Ginseng and Codonopsis and how to distinguish them to apply exactly. The main differences between Ginseng and Codonopsis are as follows.
\end{abstract}

\section{The Differences of Source}

Ginseng is the dried root and stem of the Araliaceae Ginseng (Araliace ae Panax ginseng.C.A.Meyer. Or Araliace ae Panax schinseng Nees. )

Codonopsis is the dried root of the Campanulaceae Codonopsis(Campanulaceae Codonopsis pilosula Nannfeldt).

\section{The Differences of Morphological Characteristics and Medicinal Properties}

The Differences of Morphological Characteristics. Ginseng roots are $30-60 \mathrm{~cm}$ in length, cylindrical or spindle-shaped, thick, fleshy, pale yellow-white, and there are slight branches below. The rhizome is slightly short, erect, cylindrical, green and unbranched. There are many stem top three-compound leaves on annual plants and the common name is "Three flowers". Biennial plants with a palmate compound leaf and five leaflets are named as the "Slap". The three-year-old plants with two opposite leaves of five leaflets are named as the "Two Jiazi". The four-year-old plants, with three whorled compound leaves, are named "Menorah ". The five-year-old plants are increased to four whorled compound leaves and named as the "Four leaf". The six-year-old plants with five whorled leaves in the top of their stems are named "five leaves." The scape is $30 \mathrm{~cm}$ long while umbellate inflorescence grows single in the top of the leaf, and there are dozens of flowers per inflorescence. Ginseng berry is kidney-shaped, and when it matured it is bright red.

Codonopsis root is $15-30 \mathrm{~cm}$ long, $1-3 \mathrm{~cm}$ in diameter, long and rough, spindle-shaped or 
cylindrical and the skin of the pale is sallow to brown. There are a few branches or one slightly smaller branch in the middle of the root, and on the upper, there is fine grain ring at the upper about $5-10 \mathrm{~cm}$. The twining stems are up to 2 meters or more, with the diameter of $2-4 \mathrm{~mm}$ and most branches. Leaves are opposite or bogus whorled and the petioles are approximately $0.5-2.5 \mathrm{~cm}$ long. Leaves are ovate or wide ovate, $1-6.5 \mathrm{~cm}$ long, $0.8-5 \mathrm{~cm}$ wide and the extremity of the leaves are blunt or slightly pointed. Base of the leaves are rounded truncate to shallow heart-shaped and branching leaves are gradually narrow. It is in green surface while the back surface is gray-green. Flowers are in axils of leaves solitary and petioles are nearly opposite of them. It is green, and there are stems. The lower portion of the capsule is hemispherical while the upper is conical. ${ }^{[1]}$

The Differences of Medicinal Properties. There are sparse and intermittent rough stripes and distinct longitudinal wrinkles at the upper portion or the whole Ginseng root. According to the processing methods, it can be divided into dry Radix Ginseng, white Ginseng, active Ginseng and much more. The white Ginseng is slightly harder while the dry Radix Ginseng body is slightly soaked. Both of them are with reed head, opaque section and in yellowish white. The ministry of their skin is with yellow-brown punctate resin ducts and radial cracks. It distributes rich aroma and tastes slightly bitter and sweet.

The surface of Codonopsis root is grayish-yellow, gray-brown or reddish-brown, with irregular longitudinal groove and shrinkage. The transverse lenticels are sparse, there are a lot of orbicular crimple at the upper part and they are at a particularly dense near the root of the head. The quality of Codonopsis is soft or hard and the fracture surface is smooth. It distributes micro-fragrant and tastes sweet while chewing no residue. [2]

\section{The Differences of Efficacy}

Ginseng is sweet, slightly bitter, sexual tepid. It belongs to the spleen, lung, heart and kidney meridians. There is the function of Ginseng of supplementing the promordial Qi, restoring pulse to save prostration, nourishing the spleen and lungs, resulting in Jin, nourishing the blood and tranquilizing the mind and enhancing the intelligence. Therefore it is effective medicine of supplementing Qi. It can be used for spleen deficiency, deficiency of lung Qi, body fluid injury, thirsty, symptoms of diabetes which are similar to, blood deficiency, deficiency of Qi while pathogenic Qi prevailing and other symptoms.

Codonopsis is sweet, nature. It belongs to the spleen, lung meridians. It is with the function of tonifying spleen-lung, nourishing the blood and producing fluid. It can be used for deficiency of spleen and lung Qi, injury of Qi and fluid, deficiency of Qi and blood, heat and symptoms of diabetes which are similar to and so on. Compared with Ginseng, there is not the function of supplementing the promordial Qi and restoring pulse to save prostration. [3]

\section{Differences in Usage and Dosage}

Ginseng: decocted, 3-9gram once. It can be used 15-30gram once when it is used to save the collapse with slow fire decocted. It can also be grinded into powder to swallow, 2gram once, twice a day. [4]

Codonopsis was mainly be decocted, 9-30 gram once. It can also be boiled to ointment .In addition, add it in pills or powders to swallow. [3]

\section{Differences in Chemical Composition and Pharmacological Effects}

Chemical Composition Difference. There are great deals of ingredients in Ginseng, such as Ginseng saponins, volatile oil, sugars (glucose, maltose, fructose, etc.), vitamins, amino acids, peptides, choline, spermine, cholamine, a variety of trace elements and so on. 
There are a great deal of ingredients in Codonopsis, such as saponins, trace alkaloids, sugars (sucrose, glucose, chrysanthemum sugar, etc.), starch, mucus and resins and so on.

The different types and unequal quantities of chemical composition which they contained leads to different pharmacological effects and clinical applications.

Pharmacological Effects of the Distinction. Modern pharmacological researchers have shown that Ginseng contains Ginseng saponin which could resist shock. There are the functions of Ginseng of enhancing digestion and absorption, increasing the activity of pepsin and protecting gastrointestinal cells and improving the symptoms of spleen. Besides, using Ginseng can promote the use of the body's tissues of sugar, accelerate the oxidation of sugar decomposition to provide energy for the body's tissues. Furthermore, it could promote the brain to the use of energy and enhance the ability of learning and memory. Moreover, it can also promote hematopoietic function, but also promote the anti-fatigue, anti-aging, anti-myocardial ischemia and anti-cerebral ischemia, anti-arrhythmia and so on.

There is not the function of Codonopsis of antishock, but it can be excited respiratory center. In addition, using Codonopsis can regulate gastrointestinal motility, anti-ulcer. Besides, it can also improve learning and memory, with anti-dementia puzzle role. Additionally, Codonopsis polysaccharide can adjust the imbalance of intestinal flora, while increase peripheral blood hemoglobin, spleen compensatory hematopoietic function. [5]

\section{The Difference of Clinical Application}

Ginseng :(1) Physical weakness, cold, weak pulse. There are lots of functions of Ginseng, such as supplementing the promordial Qi, restoring pulse to save prostration, and it is the most important herb to do for first aid. All sweating, vomiting, diarrhea, bleeding or serious illness caused by promordial Qi to take off, faint breath, sweating more than and the vast veins and other serious symptoms, Ginseng is available, such as Pure Ginseng Decoction. (2) The weakness of spleen and stomach, less eating, lung deficiency cough, impotence, uterine cold. This product is effective medicine of supplementing the spleen Qi, and could be applied to the people who feel weak, exhausted and eat less loose stools, such as the four gentleman Decoction.(3)The injury of Qi ,thirsty, heat and symptoms of diabetes which are similar to . It is applied to the people who injured of Qi and Jin which was all the normal liquid of the body in general. Doctors could use it with Ophiopogon, Astragalus and other herbs together. (4) For first aid, such as cardiogenic shock first aid, the combination of Ginseng and Aconite can cure death collapse. (5) Deficiency of heart $\mathrm{Qi}$, palpitation and insomnia. This product can benefit the heart Qi, soothe the nerves puzzle. It is suitable for the deficiency of heart Qi, palpitations, shortness of breath, insomnia, forgetfulness. It is often used with Astragalus, Poria, the jujube kernel and other drugs. When there is disharmony of heart kidney, deficiency of yin and bolld, insomnia, palpitations, it could use the Tianwang Buxin Pill. (6) Treatment of cardiovascular diseases. The use of ginseng can reduce the clinical symptoms of hypertension, myocardial malnutrition, coronary arteriosclerosis, angina, etc. Low dose can rise high blood pressure while high dose to reduce blood pressure. (7) Treatment of stomach and liver diseases. For patients with chronic gastritis with gastric acid deficiency and low gastric acid, taking Ginseng can make the volume of stomach increased, and the symptoms relieved or disappeared. As for patients with acute infectious hepatitis, there is a certain significance of taking Ginseng to prevent it into a chronic hepatitis, in some certain treatment conditions. (8) Treatment of neurasthenia. Using Ginseng has a significant effect on the nervous system, such as it can improve the activity of the body, reduce the body fatigue. And there is a certain effect on a variety of different types of neurasthenia. (9) Anti-tumor. Ginseng is sensitive to specific tumor cells, and it can be combined with some other drugs directly or assisted the body itself to against tumor cells` proliferation and differentiation, so as to play a role in killing tumor cells. [6][7]

On the application of the pros and cons of Ginseng, the Qing Dynasty famous doctor Lingtai Xu 
related and analyzed it in its "Shen Nong's Herbal Classic of one hundred kinds of record" in greater detail. It said: "Pharmacological effect of ginseng is powerful, if the use of ginseng is not appropriate; it is more damage for the human body than other drugs. Nowadays, doctors who use Gingseng, can cure a minority, at the same time, leading to the death of the majority of patients. The patient died from physical debilitation accounted for one-tenth to two-tenths, while the patient died of disease accounted for eight-tenths to nine-tenths. Ginseng specializes in nourishing the weak body and is not good at conquering the disease[8]. Whether or not the pathogens have been wiped out, sickness or institutional weakness for a long time, or wealth, doctors use Ginseng for sure. There are two reasons, one is too cautious, one to plug responsibility, and patients with family members believe that the use of Ginseng is in filial piety. People do not know that the pathogen has not completely dispel, then using Ginseng, as a result, it can not make enough strength to make the body much stronger, and for the deep-rooted disease there is no therapeutic effect, and ultimately it can not be cured. "Thus, the application of ginseng must be taken seriously, be dialectical to treatment and do not blindly. [9][10]

Codonopsis: (1) Deficiency of spleen and lung Qi, eating less and fatigue, cough and virtual asthma. There is the function of tonifying spleen and lung Qi of Codonopsis, which is similar to Ginseng. However, the pharmacological effect is weaker than Ginseng's. With regard to the treatment of lung Qi deficiency cough, shortness of breath, low sound lazy words and other symptoms, it could use Codonopsis with Astragalus, Gecko and other herbs to tonifying lung Qi and relieving asthma and cough. As to treat deficiency of spleen and lung Qi of the mild symptoms, it often uses the Codonopsis to replace the ancient Ginseng. (2) Lacking of blood, pale complexion, palpitations and shortness of breath. This product has the power to nourishing Qi and blood. It is suitable for the syndrome that Qi is deficient to not producing blood, or blood is deficient to not producing Qi, which caused the deficiency of both Qi and blood with pale or sallow complexion, fatigue, dizziness, palpitation. It is often used with Astragalus, Angelica, Rehmannia and other drugs in order to enhance the effect of replenishing Qi and blood. (3) Injury of Qi and Jin, shortness of breath, thirsty and symptoms of diabetes which are similar to. This product has the effect of nourishing Qi and Jin and it could be used with Radix, Schisandra, Astragalus and other drugs together. (4) It can be used in the treatment of thrombocytopenic purpura, and there is a certain effect on leukemia and thrombocytopenia. Furthermore it is often used with astragalus, licorice and other herbs. (5) It can be used to prevent and cure coronary heart disease. Codonopsis liquid can reduce the preejection period left ventricular ejection time ratio, enhance heart function, inhibiting platelet adhesion and aggregation. (6) The treatment of gastric ulcer, chronic atrophic gastritis, vomiting, etc.

The pharmacological effect of Codonopsis is more relaxation than the pharmacological effect of Ginseng. Ancient prescriptions for the treatment of lacking of lung Qi, lacking of Jin and thirsty, symptoms of diabetes which are similar to, blood deficiency and Qi deficiency and other symptoms milding and chronic disease patients, Codonopsis can be used in large doses instead of Ginseng, as for severe and acute symptoms, Ginseng is appropriate. As Codonopsis could not be used to supplementing the Qi or saving prostration, Ginseng takes the place of Codonopsis to cure the symptoms of shorting of buoyancy and collapsing. In addition, Ginseng is also good at supplementing Qi and help Yang, soothing the nerves by wisdom, but this kind of role is not obvious at Codonopsis. ${ }^{[5]}$ As of Modern physicians Shanlei Zhang said in the book which was named "Materia Medica of justice", " Codonopsis can nourish the spleen and stomach, nourish the lung and help it produce Jin, nourish the body of the gas. And there is not much differences with Ginseng and Codonopsis... The strength of Codonopsis is weak slightly and it cannot be sustained. After all the serious illness, the body is weak, taking only a little weight once, it is enough to vibrate the spirit of the body, it is really in line with the scale of the regulations, it cannot be sustained. However, as for nourishing vitality and nourishing other organs, they are all ancient and modern prescriptions which used Ginseng there cannot be replaced with Codonopsis. "Therefore, using Codonopsis instead of Ginseng should be dialectical. [11]

In summary, there are a lot of similarities in Codonopsis and Ginseng which are from different 
families and genera, the differences in the pharmacological effects and clinical application should still be cautious. Clinical medication should be on the basis of accurate grasp of the relevant information of the two drugs and to judge the hour and size up the situation and use them dialectical.

\section{References}

[1] C.J. Song, The Botanical Origin Identification of Ginseng and Codonopsis, Ginseng research. 2(2016) 62-64. (In Chinese)

[2] Editorial Committee of Chinese Journal of Plant Science, Chinese Academy of Sciences, Flora of China. Science Press.(In Chinese)

[3] G.S.Zhong. China Press of Traditional Chinese Medicine.(Science of Chinese Materia Medica.China 2012). (In Chinese)

[4] Q.Y.Sun, The Difference between Ginseng and American Ginseng, Chinese Journal of Practical Medicine, 15(2013) 243-243. (In Chinese)

[5] J.Wang, C.Q.Deng and L.Shi, etc. Modern Research Progress of Codonopsis Pilosula, Guide of China Medicine, 31(2011) 279-281. (In Chinese)

[6] Y. Feng, Research Progress on Pharmacological Action and Clinical Application of Ginseng, Clinical Research of TCM, 6(2013) 121-122. (In Chinese)

[7] Shin B K, Kwon S W, Park J H, et al, Chemical diversity of ginseng saponins from Panax ginseng, Journal of Ginseng Research, 16(2015) 287-298.

[8] J. Wan, Y. Fan, Q. Yu, et al. Integrated evaluation of malonyl ginsenosides, amino acids and polysaccharides in fresh and processed ginseng, Journal of Pharmaceutical \& Biomedical Analysis, 107(2015) 89-97.

[9] Chung I M, Lim J J, Ahn M S, et al. Comparative Phenolic Compounds Profiles and Antioxidative Activity of the Fruit, Leaves, and Roots of Korean Ginseng ( Panax ginseng, Meyer) According to Cultivation Years, Journal of Ginseng Research, 40(2016) 68-75.

[10] Dong, Sung-II, Jang, et al, Anti-Proliferative Effects of Ginsenosides Extracted from Mountain Ginseng on Lung Cancer, Chinese Journal of Integrative Medicine, 22(2016) 344-352.

[11] Kwang-Soo B, Yi Y S, Young-Jin S, et al, In vitroandin vivoanti-inflammatory activities of Korean Red Ginseng-derived components, Journal of Ginseng Research, 40(2016) 437-444. 